\title{
UAV-BASED HYPERSPECTRAL DATA ANALYSIS FOR URBAN AREA MAPPING
}

\author{
N. A. Suran ${ }^{1}$, H. Z. M. Shafri ${ }^{1}$, *, N. S. N. Shaharum ${ }^{1}$, N. A. W. M. Radzali ${ }^{1}$, V. Kumar ${ }^{2}$ \\ ${ }^{1}$ Department of Civil Engineering and Geospatial Information Science Research Centre (GISRC), Faculty of Engineering, Universiti \\ Putra Malaysia, 43400 Serdang, Selangor, Malaysia - asyikin.suran@gmail.com, helmi@ upm.edu.my, fieranisa94@gmail.com, \\ wahidamohdradzali1992@gmail.com \\ ${ }^{2}$ ORNET Sdn. Bhd.47-4, Jalan SP 2/1, Taman Serdang Perdana, Seksyen 2,43300 Seri Kembangan, Selangor, Malaysia - \\ support@ornets.com
}

KEY WORDS: Airborne, UAV, Hyperspectral, Urban Area, Artificial Neural Network, Support Vector Machine

\begin{abstract}
:
A recent development in low-cost technology such as Unmanned Aerial Vehicle (UAV) offers an easy method for collecting geospatial data. UAV plays an important role in land resource surveying, urban planning, environmental protection, pollution monitoring, disaster monitoring and other applications. It is a highly adaptable technology that is continuously changing in innovative ways to provide greater utility. Thus, this study aimed to evaluate the capability of UAV-based hyperspectral data for urban area mapping. In order to do the mapping, Artificial Neural Network (ANN), Support Vector Machine (SVM), Maximum Likelihood (ML) and Spectral Angle Mapper (SAM) were used to classify the urban area. The classifications involved seven classes: concrete, aluminium, flexible pavement, clay tile, interlocking block, tree and grass. Then, the overall accuracies obtained from ANN, SVM, ML and SAM for $0.3 \mathrm{~m}$ spatial resolution images were $92.33 \%, 85.86 \%, 83.41 \%$ and $46.55 \%$ with the kappa coefficient of $0.91,0.83,0.80$ and 0.38 respectively. Thus, the classification results showed that the powerful and intelligent ANN algorithm produced the highest accuracy compared to the other three algorithms. Overall, mapping of urban area using UAV-based hyperspectral data and advanced algorithms could be the way forward in producing updated urban area maps.
\end{abstract}

\section{INTRODUCTION}

The advances in technology in the scientific field of remote sensing (RS) have attracted the interest of several sciences, including civil engineering, covering from the basic principles, methods and applications in which, can provide a good source of useful information for a wide range of issues. Civil engineering is a broad field that consists of many sub-divisions such as surveying, construction, transportation, water resources, environmental and coastal. RS offers methods on several applications that can be used in the main areas of civil engineering such as urban planning, site investigation, terrain mapping, road and highway construction, the study of natural hazards and environmental issues, monitoring and others. RS is one of the methods that can be used to collect geospatial data over large areas in a short time and cost-efficient. It plays an important role in providing required near-real-time images or data that can potentially help civil engineers to plan and make decisions effectively.

There are two types of sensors used in RS which are active and passive sensors. Passive sensor can acquire two types of data namely multispectral and hyperspectral data. By comparing to multispectral data, hyperspectral data are more robust because the data consist of subtle information due to a higher number of bands provided.

Hyperspectral data generally contain between 100 to 200 or more spectral bands with narrow bandwidth, compared to multispectral data which provide from 5 to 10 spectral bands (Kumar \& Yarrakula, 2017; Taherzadeh \& Shafri, 2011). The high spectral resolution provided in hyperspectral RS data has introduced great potential to analyse complex urban scenes.
Mapping urban area is challenging due to the wide range of spectral signatures or combination with the occurrence of mixed pixels. It can be improved through the accurate spatial resolution, improved classification algorithm and the use of satellite imagery with high spatial and spectral resolution (Obade \& Paul, 2007). Lu \& Weng (2006) have showed the limitations of using multispectral sensors for the extraction of urban impervious surfaces compared to the capabilities of hyperspectral sensor. The study found that hyperspectral data provide many advantages compared to conventional multispectral data in detecting impermeable surfaces in urban areas. Hyperspectral data are unique and easy to discriminate against a wide variety of natural and human-made materials (Deshpande, Inamdar, \& Vin, 2013).

UAV is a type of vehicle used to collect RS data through the airborne platform. It is inexpensive, easy to use and furthermore, the user has an upper hand in the decision making for selecting the place, flying height(altitude) and the flying time. These options will help the user to define the desired spatial resolution and the best time to fly the UAV (Noor, Abdullah, \& Hashim, 2018). Hence, the utilisation of the UAV system to acquire geospatial data showed a great interest in several mapping applications in recent years.

UAV system can collect high-resolution RS data on small areas in a short period, and it is less expensive compared to manned aerial surveying. UAV's system has been used in numerous applications such as slope mapping (Yusoff et al., 2018), agricultural (Gay et al., 2009), fire monitoring (Zhouet al., 2005) and vehicle detection (Kaaniche et al., 2005). However, the capability of UAV-based hyperspectral data for urban area in Malaysia is yet to be explored. Therefore, the aim of this paper is to evaluate the capability of UAV-based hyperspectral data over an urban area in Universiti Putra Malaysia (UPM). 


\section{STUDY AREA}

The data for this study were collected at the Main Campus, UPM, Serdang which is located south of Kuala Lumpur between $3^{\circ} 00^{\prime} 00^{\prime \prime} \mathrm{N}$ and $101^{\circ} 42^{\prime} 25^{\prime \prime}$ E. (Fig 1)

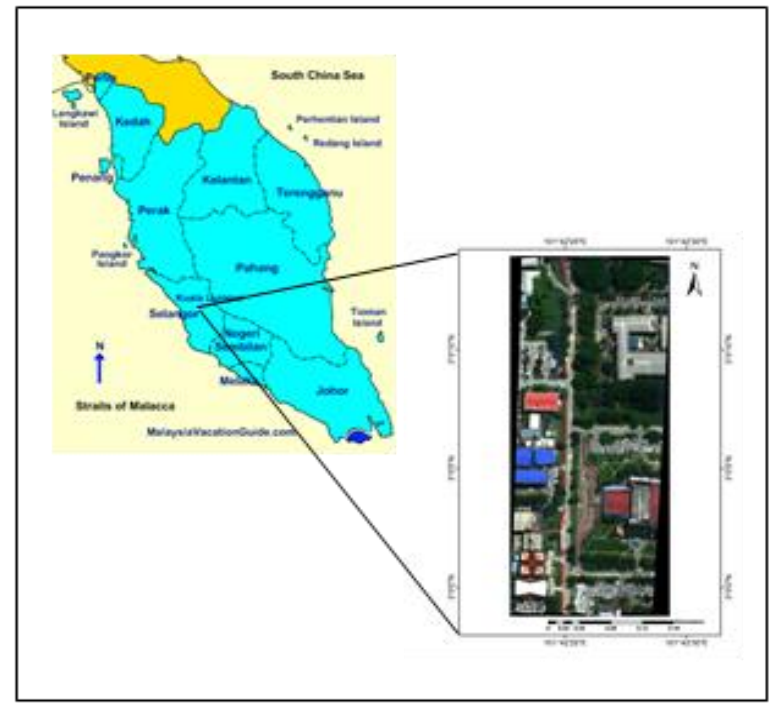

Figure 1: Study area and acquired data.

\section{METHODOLOGY}

The research was conducted based on the methodology flowchart as shown in Figure 2.
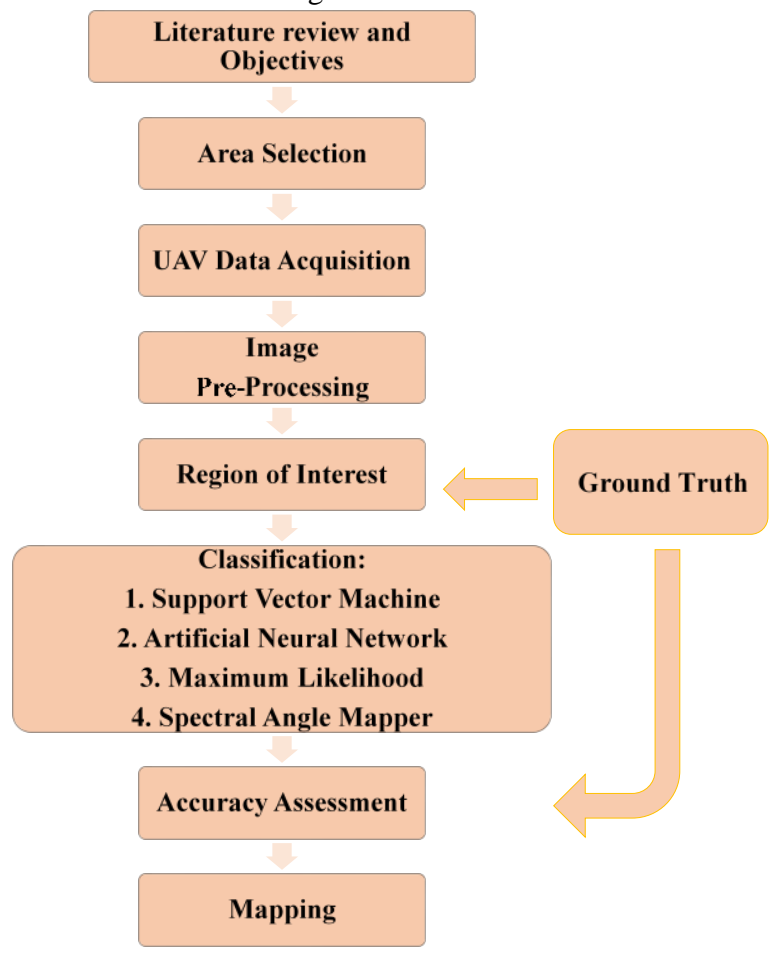

Figure 2: Flow of Work

\subsection{Data Acquisition}

Data collection was done in March 2018 at a test area in UPM, Selangor by using a hexacopter UAV. The data were acquired using Bayspec OCI-F hyperspectral sensor. Table 1 detailed out the specifications of the sensor. A total of 116 bands were obtained ranging from 400 to $1000 \mathrm{~nm}$ with a spatial resolution of $0.3 \mathrm{~m}$. The UAV was set to fly at a $160 \mathrm{~m}$ of the altitude above ground level with $21^{\circ}$ field of view. Figure 3 shows the $\mathrm{UAV}$ and hyperspectral sensor set-up for the data acquisition.

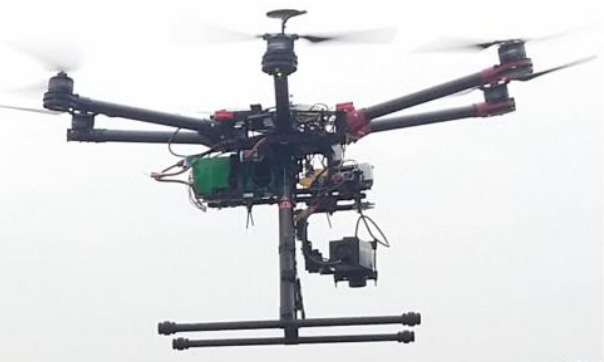

Figure 3: The UAV platform with the hyperspectral sensor onboard.

Table 1: Bayspec OCI-F sensor specifications

\begin{tabular}{|l|l|}
\hline \multicolumn{2}{|c|}{ Key Specifications } \\
\hline Operation Mode & "True push-broom" \\
\hline Spectral Range & $400-1000 \mathrm{~nm}$ or 900-1700 nm \\
\hline $\begin{array}{l}\text { Number of Spectral } \\
\text { Bands }\end{array}$ & Up to 240 (VIS-NIR) / 80 (SWIR) \\
\hline Spatial Pixels & $\begin{array}{l}800 \mathrm{x} \text { scan length (VIS-NIR), 250 x } \\
\text { scan length (SWIR) }\end{array}$ \\
\hline Lens & $16 \mathrm{~mm}\left(21^{\circ}\right.$ FOV) \\
\hline
\end{tabular}

\subsection{Ground Truth}

Ground truth is referred to the data collected on targeted field. The collection of measurements and observations relating to the type, size, condition and other important physical or chemical properties of materials on the surface of the earth is one of the most important elements of ground truth. To do this, field survey has been done in order to validate the output produced by checking the type of material on the main campus area of UPM and finally 7 classes were defined to generate ground truth map.

\subsection{Development of Training Samples}

The training samples for this study were created using Region of Interest (ROI) in ENVI software based on selected features. Seven classes were created namely concrete, aluminium, flexible pavement, clay tile, interlocking block, tree and grass. Each class was assigned with a certain number of ROIs and colour. Training and testing pixels were created based on random sampling. The training and testing pixels for the classified classes were provided in Table 2 and Figure 3.

Table 2: Training and testing pixels used for image classification

\begin{tabular}{|l|c|c|}
\hline \multicolumn{1}{|c|}{ Classes } & Training Pixels & Testing Pixels \\
\hline Concrete & 5249 & 2287 \\
\hline Aluminium & 4411 & 1913 \\
\hline Flexible Pavement & 4617 & 2117 \\
\hline Clay Tile & 505 & 231 \\
\hline Interlocking Block & 890 & 405 \\
\hline Tree & 11400 & 4940 \\
\hline Grass & 2808 & 1243 \\
\hline
\end{tabular}




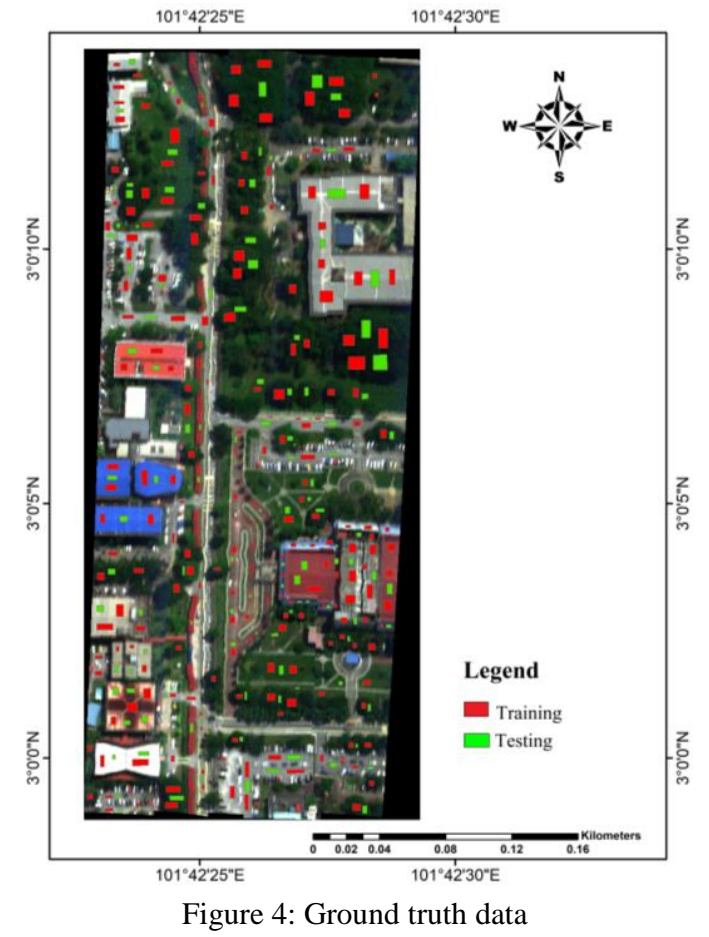

\subsection{Algorithms Used in This Study}

This study used ENVI 5.3 software to classify and compare the results produced from some standard machine learning and nonmachine learning algorithms.

\subsubsection{Artificial Neural Network (ANN)}

The ANN is widely used for the analysis of RS images. The advantages of an ANN model include the ability to solve non linear relationships, no underlying data assumption, the integration of a priori knowledge and the ability to incorporate various data types into the analysis. The neural network in ANN structure has a key element where it can learn on its own. The algorithm not only a complicated system, but it is also a complex system where the information structure flowing through its network can be changed or shifted. However, it can be accomplished by adjusting the connection weight. Every connection in the system has a parameter called weight.

Weight is a number that controls the signal between two neurons. The weight adjustment is not required if the output produced is excellent. In contrast, a poor output will lead to error, and weight needs to be adjusted to improve the results (Bala \& Kumar, 2017). A number of trials using different parameters value which involved training threshold contribution, training rate, training momentum and training RMS exit criteria were carried out. Hence, the combination of values that produced the best output was used in this study, in which the values were $0.7,0.4,0.7$ and 0.3 for training threshold contribution, training rate, training momentum and training RMS exit criteria respectively.

\subsubsection{Support Vector Machine (SVM)}

SVM is a non-parametric supervised classification which resulted from arithmetic learning theory. This training algorithm records the training information into a higher dimensional spaces. Besides, classes can be separated by using ideal hyperplanes with small classification errors. The ideal hyperplane located using training samples that produce the highest margin are called support vectors. This algorithm can be used on high-dimensional data with a small size of training sample (Taylor, Pal, \& Mather, 2006). One advantage of using SVM is high accuracy can be obtained even with limited number of training samples (Zheng et al., 2015). It is because SVM consists of kernels that can transform the data into the required form. Therefore, this study utilised one of the most promising kernels, Radial Basis Function (RBF) with a gamma value of 1.0 to perform the classification.

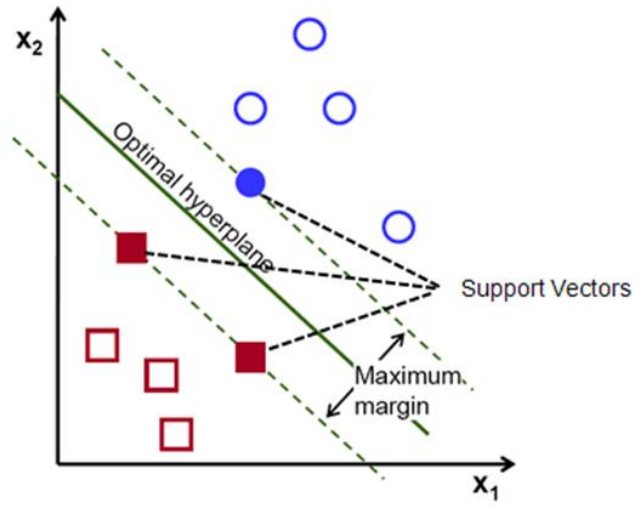

Figure 5: Hyperplane in SVM

\subsubsection{Maximum Likelihood (ML)}

ML classification is one of the most common supervised classification techniques used with RS image data. Due to its availability in any image processing software, the ML is a wellknown parameter algorithm. This algorithm works by assuming the statistic for each class are normally distributed in each band and the probability of a pixel to a particular class is calculated. Each pixel is allocated to the highest probability class. In this study, before the best output can be determined, different values of probability threshold were tested to classify the image, and it was found that the outputs produced for this data were likely the same. However, if the highest probability is lower than the specified threshold, the pixel will not be classified (Park, 2016).

\subsubsection{Spectral Angle Mapper (SAM)}

SAM is a physically based spectral classification that applies an angle of n-dimensional to perform the pixels with reference spectra. This method is insensitive to lighting because SAM algorithm only uses the vector direction without including the length of the vector. Wang et al. (2015) stated that this technique assumes that the data had already been reduced to apparent reflectance where all the dark current and path radiance biases are removed.

The algorithm identifies the similarity between two spectra by calculating the angle between the spectra labeling them as a vector. The lines that connecting spectrum point and the origin consisted of possible material position. It will cause poorly illuminated pixels if it falls closer to the origin than pixels with a similar spectral signature but greater illumination. The value of maximum angle radians is sensitive, thus can affect the outcome. This study used a value of 100 for the aforementioned parameter and pixels beyond the stated maximum angle threshold in radians will not be classified (Kruse et al., 1993). 


\subsection{Accuracy Assessment}

In remotely sensed data, the term of accuracy is used to show the degree of correctness of a classified map. A confusion matrix was used to calculate the accuracy of the classification result. The dataset used in this study was divided into $70 \%$ training dataset to classify the image and $30 \%$ test dataset to validate the output produced in terms of classified maps.

\section{RESULTS AND DISCUSSIONS}

Table 3: Accuracy assessment for ANN, SVM, ML and SAM

\begin{tabular}{ccc}
\hline Classifier & $\begin{array}{c}\text { Overall } \\
\text { accuracy }(\%)\end{array}$ & $\begin{array}{c}\text { Kappa } \\
\text { Coefficient }\end{array}$ \\
\hline ANN & 92.33 & 0.91 \\
\hline SVM & 85.86 & 0.83 \\
\hline ML & 83.41 & 0.80 \\
\hline SAM & 46.56 & 0.38 \\
\hline
\end{tabular}

The results of classification in terms of accuracy results and classification maps are given in Table 3 and Figures 5 to 8 respectively.

The result obtained showed that machine learning techniques which were ANN and SVM produced higher accuracy with $92.33 \%$ and $85.86 \%$ for overall accuracy with the kappa coefficient of 0.91 and 0.83 respectively. Compared to nonmachine learning techniques, SAM and ML produced lower accuracy with $46.55 \%$ and $83.41 \%$ for overall accuracy with a kappa coefficient of 0.38 and 0.80 respectively.

Even though ML classification is a non-machine learning technique, the result produced is reasonable because the visualisation and the accuracy produced were almost similar to the result produced by SVM. However, by comparing the results obtained with the collected ground truth data, it showed that SVM produced better output compared to ML although SVM and ML had a slight difference in terms of accuracy.

Among the four algorithms, SAM produced the worst image classification. The setting of the maximum angle threshold has a major impact on the classification results (Tan \& Hou, 2016). The algorithm can only classify well for clay tile class. From Figure 9, it showed that most of the pixels were misclassified. Some of the aluminium pixels were misclassified to clay tile and interlocking block. From Figure 13, it shows that SAM misclassified the aluminium into flexible pavement and concrete. For ML, the algorithm can classify well for tree and grass classes. From figure 12, the result produced showed that the aluminium pixels were misinterpreted as the combination of flexible pavement and concrete. Meanwhile, in Figure 11, SVM produced a better output compared to ML where most of the aluminium pixels were detected with the presence of flexible pavement in a small amount. This algorithm was able to classify grass, tree and clay tile pixels. Some of the flexible pavement pixels at $3^{\circ} 0^{\prime} 10^{\prime \prime} \mathrm{N}$ on the left area misinterpreted as aluminium and some of concrete roof at $3^{\circ} 0^{\prime} 0^{\prime} ' \mathrm{~N}$ on the left area were misclassified into aluminium and flexible pavement. For ANN, the algorithm can classify well for grass, tree and clay tile pixel. Some classes such as roof materials at $3^{\circ} 0^{\prime} 0^{\prime \prime} \mathrm{N}$ on the left area which is concrete pixels were mixed with aluminium. From Figure 10, the algorithm has correctly detected the aluminium pixels and classified similarly to the ground truth.
Thus, this finding showed that machine learning algorithm is more intelligent compared to non-machine learning algorithm because the machine learning algorithms such as SVM and ANN have a greater number of parameters to be used in classification compared to SAM and ML. Besides producing the highest overall accuracy, ANN classified the best for most of the classes and thus, ANN proved to be the best algorithm of the four methods tested. This is because of ANN can perform the artificial function on how the human brain works. Besides that, ANN has greater number of parameters compared to SVM where it increased the accuracy of the results.

Table 4: Confusion matrix of ANN

\begin{tabular}{|l|l|l|l|l|l|l|l|}
\hline Classes & A & B & C & D & E & F & G \\
\hline A & 2091 & 271 & 99 & 1 & 0 & 0 & 22 \\
\hline B & 119 & 1623 & 39 & 0 & 0 & 0 & 6 \\
\hline C & 57 & 16 & 1979 & 1 & 0 & 0 & 72 \\
\hline D & 15 & 1 & 0 & 229 & 0 & 0 & 0 \\
\hline E & 0 & 2 & 0 & 0 & 1190 & 12 & 0 \\
\hline F & 1 & 0 & 0 & 0 & 53 & 2102 & 0 \\
\hline G & 4 & 0 & 0 & 0 & 0 & 0 & 305 \\
\hline
\end{tabular}

Note: A, Concrete; B, Aluminium; C, Flexible Pavement D, Clay Tile; E, Grass; F, Tree; G, Interlocking Block.

Table 5: Confusion matrix of SVM

\begin{tabular}{|l|l|l|l|l|l|l|l|}
\hline Classes & A & B & C & D & E & F & G \\
\hline A & 2010 & 249 & 225 & 2 & 64 & 0 & 0 \\
\hline B & 117 & 1402 & 239 & 0 & 4 & 0 & 0 \\
\hline C & 105 & 262 & 1652 & 0 & 68 & 0 & 0 \\
\hline D & 1 & 0 & 0 & 229 & 0 & 0 & 0 \\
\hline E & 45 & 0 & 1 & 0 & 269 & 0 & 0 \\
\hline F & 0 & 0 & 0 & 0 & 0 & 1196 & 20 \\
\hline G & 9 & 0 & 0 & 0 & 0 & 47 & 2094 \\
\hline
\end{tabular}

Note: A, Concrete; B, Aluminium; C, Flexible Pavement; D, Clay Tile; E, Interlocking Block; F, Grass; G, Tree.

Table 6: Confusion Matrix of ML

\begin{tabular}{|l|l|l|l|l|l|l|l|}
\hline Classes & A & B & C & D & E & F & G \\
\hline A & 1003 & 62 & 5 & 0 & 0 & 79 & 8 \\
\hline B & 493 & 1827 & 65 & 0 & 0 & 154 & 0 \\
\hline C & 36 & 60 & 269 & 0 & 0 & 7 & 0 \\
\hline D & 0 & 0 & 0 & 1209 & 18 & 0 & 0 \\
\hline E & 0 & 0 & 0 & 340 & 2096 & 10 & 0 \\
\hline F & 381 & 168 & 66 & 0 & 0 & 2015 & 42 \\
\hline G & 0 & 0 & 0 & 0 & 0 & 22 & 181 \\
\hline
\end{tabular}

Note: A, Aluminium; B, Flexible Pavement; C, Interlocking Block; D, Grass; E, Tree; F, Concrete; G, Clay Tile. 
The International Archives of the Photogrammetry, Remote Sensing and Spatial Information Sciences, Volume XLII-4/W16, 2019 6th International Conference on Geomatics and Geospatial Technology (GGT 2019), 1-3 October 2019, Kuala Lumpur, Malaysia

Table 7: Confusion matrix of SAM

\begin{tabular}{|l|l|l|l|l|l|l|l|}
\hline Classes & A & B & C & D & E & F & G \\
\hline A & 398 & 189 & 200 & 0 & 36 & 0 & 0 \\
\hline B & 27 & 0 & 52 & 9 & 0 & 0 & 0 \\
\hline C & 442 & 1068 & 1701 & 0 & 83 & 0 & 0 \\
\hline D & 97 & 421 & 4 & 222 & 0 & 13 & 0 \\
\hline E & 1323 & 235 & 160 & 0 & 286 & 0 & 0 \\
\hline F & 0 & 0 & 0 & 0 & 0 & 1114 & 164 \\
\hline G & 0 & 0 & 0 & 0 & 0 & 987 & 1079 \\
\hline
\end{tabular}

Note: A, Concrete; B, Aluminium; C, Flexible Pavement; D, Clay Tile; E, Interlocking Block; F, Tree; G, Grass.

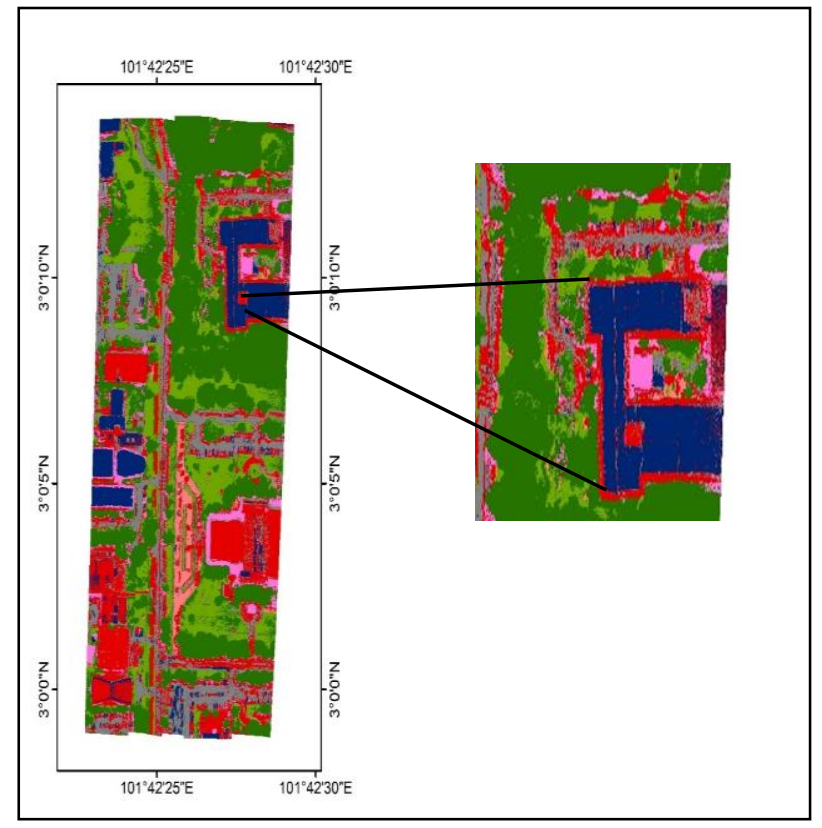

Figure 6: Figure of ANN

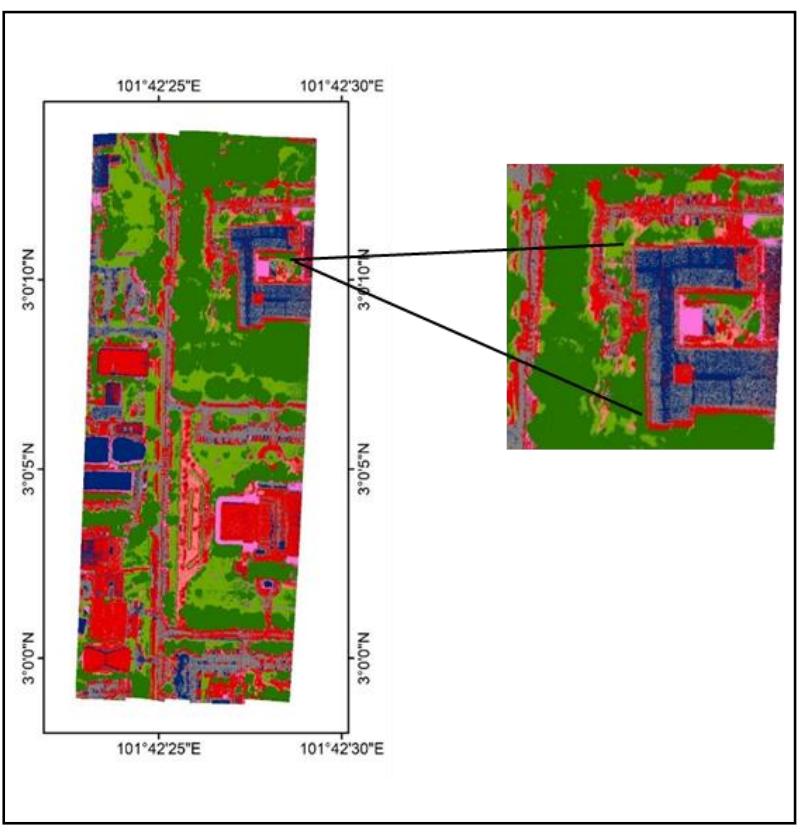

Figure 7: Figure of SVM

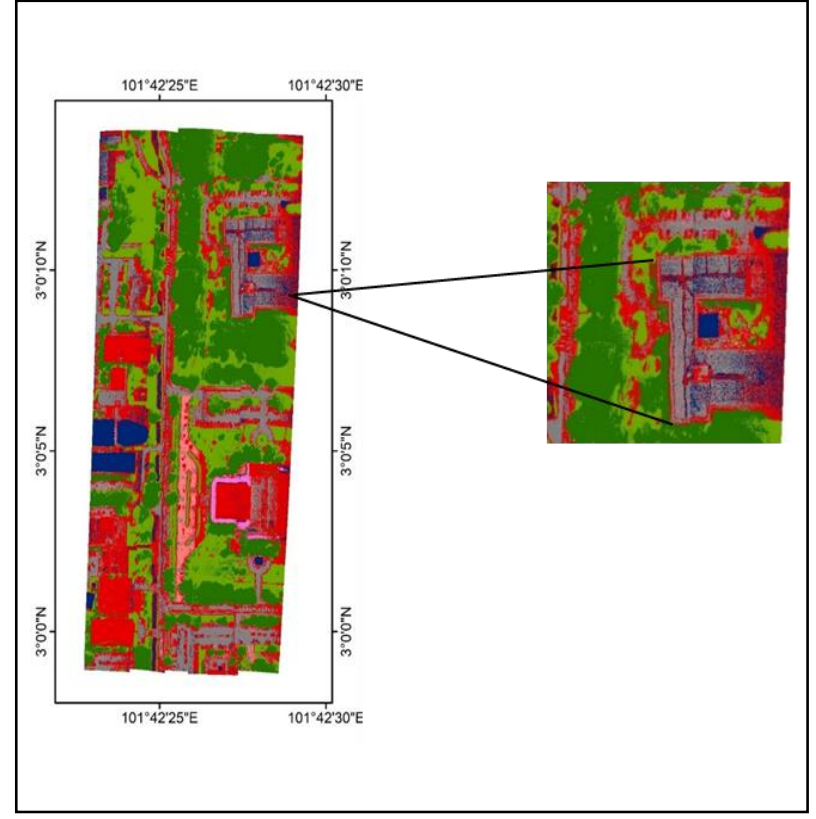

Figure 8: Figure of ML

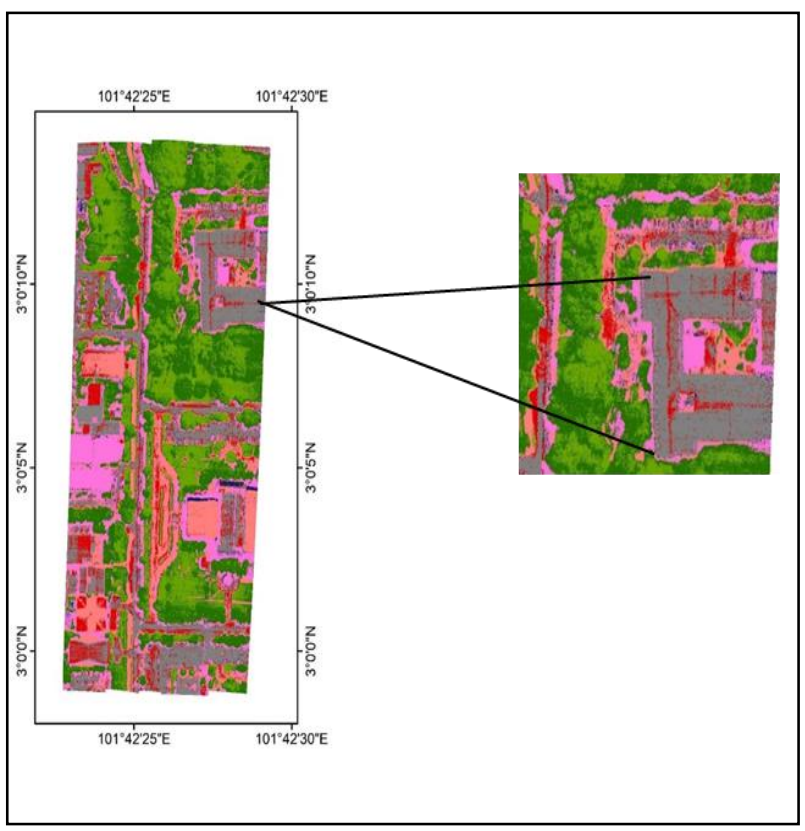

Figure 9: Figure of SAM

\section{CONCLUSION}

This study demonstrated the potential of using UAV-based hyperspectral remote sensing data in mapping urban area. The urban classification accuracy of hyperspectral data was evaluated using ANN, SVM, ML and SAM. The results showed that ANN outperformed the others by obtaining the highest overall accuracy and kappa coefficient. ANN is an effective method of machine learning to detect urban area compositions from RS data. Besides producing an accurate result, ANN algorithm is more advantageous compared to the other algorithms due to the fact that it has many parameters capability to work similarly like the neuron in human brain. Therefore, this study has shown that the use of UAV-based hyperspectral data has a great potential for mapping urban area. 
Future work can be done in order to improve the results obtained such as using other machine learning algorithms or more advanced approach such as deep learning. Besides that, the classification also can be tested using different environment such as Python programming.

\section{ACKNOWLEDGEMENT}

The authors acknowledge the facilities and financial supports provided by Universiti Putra Malaysia (UPM) via the research grant UPM/700-1/2/GPB/2017/9543100. Comments from anonymous reviewers in improving this paper are highly appreciated.

\section{REFERENCES}

Bala, R., \& Kumar, D. (2017). Classification Using ANN : A Review, 13(7), 1811-1820.

Deshpande, S., Inamdar, A., \& Vin, H. (2013). Overview of Hyperspectral Remote Sensing of Impervious Surfaces in Urban Environment, (January), 128-140. https://doi.org/10.1007/9783-642-45025-9

Gay, A. P., Stewart, T. P., Angel, R., Easey, M., Adrian, J., Thomas, N. J., \& Kemp, A. I. (2009). Developing Unmanned Aerial Vehicles for Local and Flexible Environmental and Agricultural Monitoring, (September), 8-11.

Kaaniche, K., Champion, B., Pegard, C., \& Vasseur, P. (2005). A Vision Algorithm for Dynamic Detection of Moving Vehicles with a UAV *, (April), 78-83.

Kruse, F. A., Lefkoff, A. B., Boardman, J. W., Heidebrecht, K. B., Shapiro, A. T., Barloon, P. J., \& Goetz, A. F. H. (1993). The Spectral Image Processing System (SIPS) Interactive Visualization and Analysis of Imaging Spectrometer Data, 163(October 1992), 145-163.

Kumar, M. V., \& Yarrakula, K. (2017). Comparison of efficient techniques of hyper-spectral image preprocessing for mineralogy and vegetation studies, 46(May), 1008-1021.

Noor, N. M., Abdullah, A., \& Hashim, M. (2018, June). Remote sensing UAV/drones and its applications for urban areas: a review. In IOP Conference Series: Earth and Environmental Science (Vol. 169, No. 1, p. 012003). IOP Publishing

Obade, B. Y., \& Paul, V. D. E. (2007). Remote Sensing: New Applications for Urban Areas (Vol. 95, pp. 2267-2268).

Park, B. (016). Quality Evaluation of Poultry Carcass. Computer Vision Technology for Food Quality Evaluation. Elsevier Inc. https://doi.org/10.1016/B978-0-12-802232$0.00009-8$

Taherzadeh, E., \& Shafri, H. Z. M. (2011). Using Hyperspectral Remote Sensing Data in Urban Mapping Over Kuala Lumpur, (April). https://doi.org/10.1109/JURSE.2011.5764805

Taylor, P., Pal, M., \& Mather, P. M. (2006). International Journal of Remote Support vector machines for classification in remote sensing, (March 2013), 37-41. https://doi.org/10.1080/01431160512331314083

Wang, K., Yong, B., Gu, X., Xiao, P., \& Zhang, X. (2015). Spectral Similarity Measure Using Frequency Spectrum for Hyperspectral Image Classification, 12(December 2014). https://doi.org/10.1109/LGRS.2014.2329183

Yusoff, A. R., Darwin, N., Majid, Z., Ariff, M. F. M., Idris, K. M., Vi, C., \& Vi, W. G. (2018). Comprehensive Analysis of Flying Altitude for High Resolution Slope Mapping Using Uav Technology, XLII(March), 18-21.

Tan, L., \& Hou, M. (2016). A study on the application of SAM classification algorithm in seal of calligraphy and painting based on hyperspectral technology. 2016 4th International Workshop on Earth Observation and Remote Sensing Applications (EORSA), 415-418. https://doi.org/10.1109/EORSA.2016.7552841

Zheng, B., Myint, S. W., Thenkabail, P. S., \& Aggarwal, R. M. (2015). International Journal of Applied Earth Observation and Geoinformation A support vector machine to identify irrigated crop types using time-series Landsat NDVI data. International Journal of Applied Earth Observations and Geoinformation, 34, 103-112. https://doi.org/10.1016/j.jag.2014.07.002

Zhou, G., Li, C., \& Cheng, P. (2005). Unmanned Aerial Vehicle ( UAV ) Real-time Video Registration for Forest Fire Monitoring. Proceedings. 2005 IEEE International Geoscience and Remote Sensing Symposium, 2005. IGARSS '05, 3(1), 1803-1806. https://doi.org/10.1109/IGARSS.2005.1526355

Revised August 2019 